\title{
Liquid Metal Sloshing for High-load Active Self-healing System: An Application to Tendon-driven Legged Robot
}

Shinsuke Nakashima ${ }^{1}$, Kento Kawaharazuka ${ }^{1}$, Yuya Nagamatsu ${ }^{1}$, Koki Shinjo ${ }^{1}$, Akihiro Miki $^{1}$, Temma Suzuki ${ }^{1}$, Yuki Asano ${ }^{1}$, Yohei Kakiuchi ${ }^{1}$, Kei Okada ${ }^{1}$, Masayuki Inaba ${ }^{1}$, and Alberto Pepe ${ }^{1}$

${ }^{1}$ Affiliation not available

February 14, 2022

\begin{abstract}
This Supporting information includes supporting fugures and videos for our paper. The information's Fig. 16 is the high-quality version of the article's Supplementary Movie 1.

Corresponding author(s) Email: nakashima@robot.t.u-tokyo.ac. jp
\end{abstract}

\section{ToC Figure}



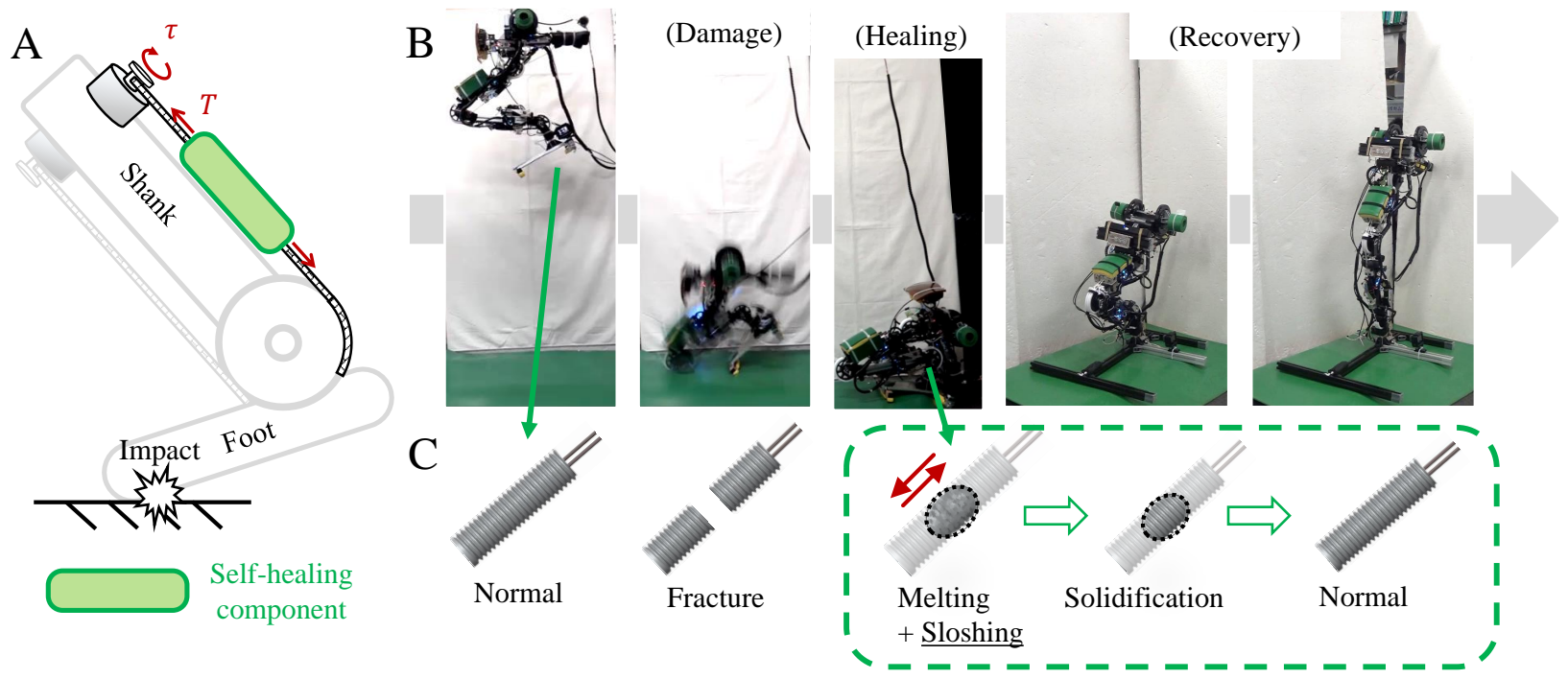

Figure 1: ToC Figure. A) Schematic of the self-healing robot. Tendon-driven legged robot has the selfhealing component on its Achilles tendon. The landing impact breaks the component, which protects other stuff on the motor-driven tendon. B) Self-healing behavior of the tendon-driven legged robot. After waiting for the self-healing sequence, the robot performed squat motions thanks to the healed strength of the selfhealing component. C) Self-healing module. The LMPA part breaks after the landing impact. The selfhealing sequence is composed of reconnection, melting and solidification. The melting process is accompanied by sloshing by the motor-driven tendon. In other words, the robot augments the self-healing performance by its own motion.

\section{Introduction}

The paper is the evolution from the authors' previous studies (Nakashima et al., 2018, 2019, 2021). We have developed several self-healing modules oriented for high-load applications such as legged robots. The module used low melting point alloy (LMPA), and the modules achieved transmission large initial tension and executed the self-healing sequence automatically. However, the modules have been suffered from the drop of the healed strength after the self-healing sequence. This study solves the problem by exploiting the robot's own actuator. Motor-driven tendon's vibration makes the liquid metal sloshing behavior which devastates the surface oxide on the fracture surface of the LMPA component. We validated the approach by the benchtop experiment and the robot's active self-healing behavior. We exploit the liquid metal sloshing caused by the robot's actuator to enhance the healed strength up to tens of kilograms. The research provides another approach, the robot's own motion, to self-healing robotics which has been improved bythe evolution of the materials, the composites, and the structures. 


\section{Supplementary Figures}

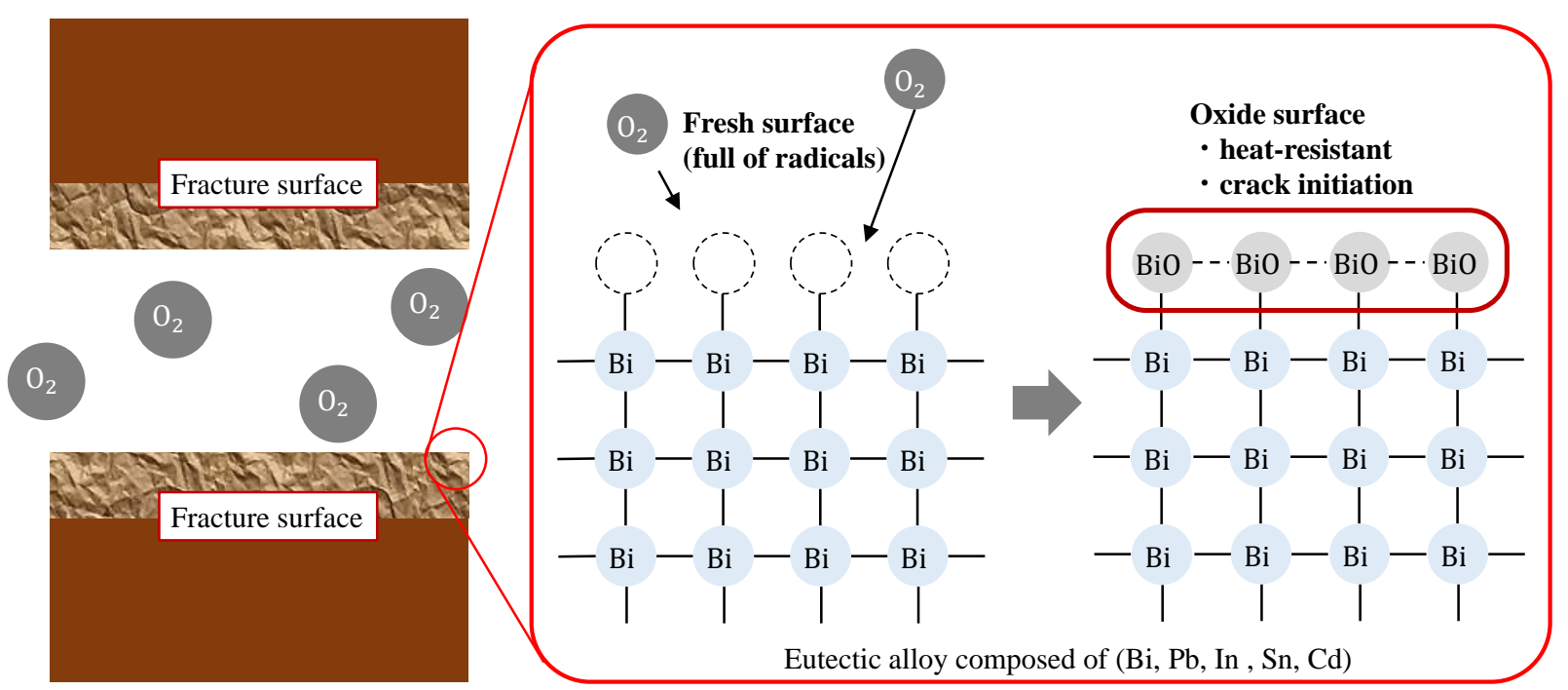

Figure 2: Schematic of the surface oxidation after the fracture. The fracture makes the fresh surface full of radicals. The oxygen molecules around the surface make the metallic oxides on the surface just after the fracture..
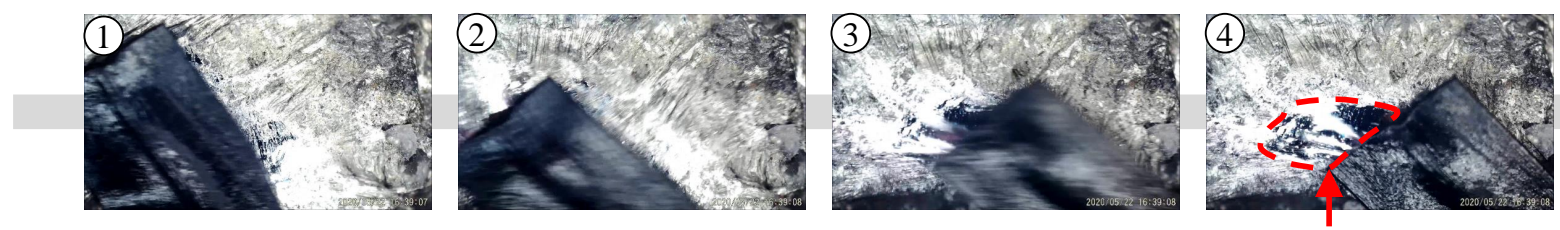

Liquid metal

Figure 3: Surface oxide on liquid metal. The traceroute of the stick shows a shiny metallic surface. Other areas show dull-white areas. 

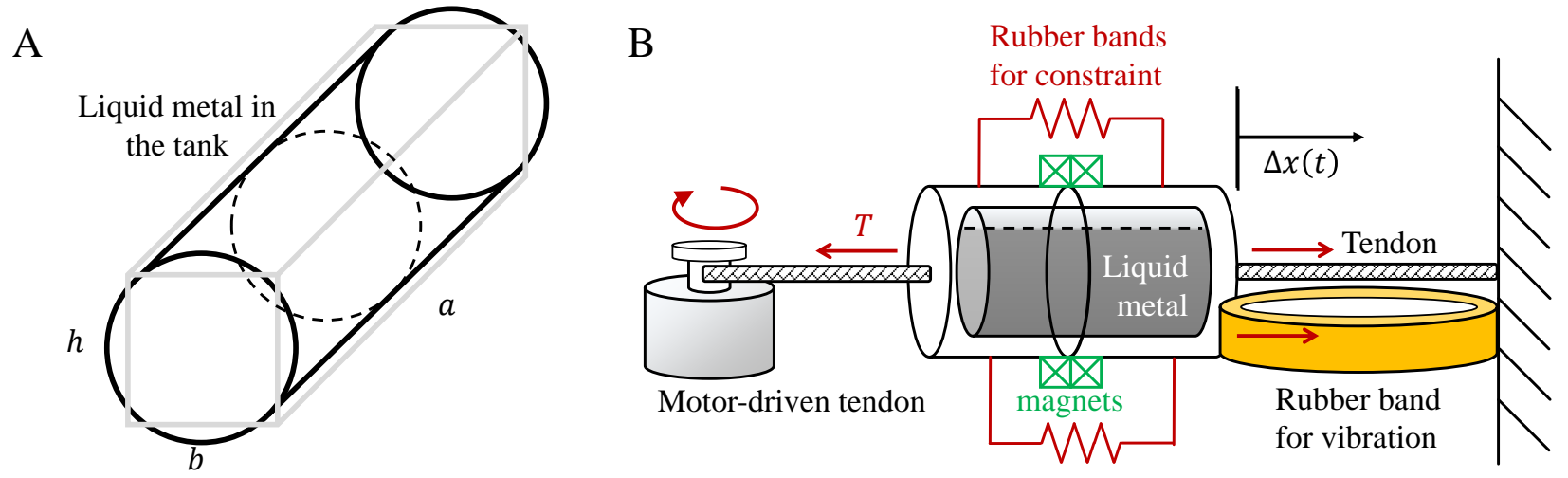

Figure 4: Schematic of sloshing validation experiment. A) The calculation process of the resonant frequency assumes that the self-healing module's shape is a cuboid. B) A motor-driven tendon is not able to generate thrust force. There-fore, the rubber band is necessary for generating the module's vibration.

A
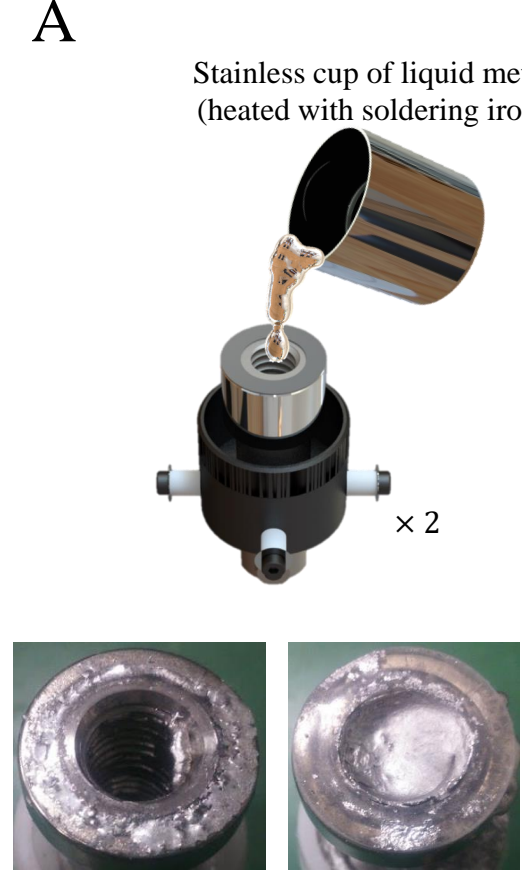

Empty

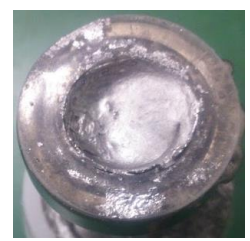

LMPA filled
B

Attaching the two magnets

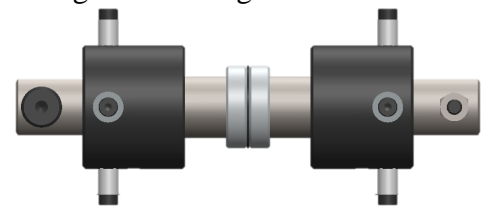

Installing the rubber bands

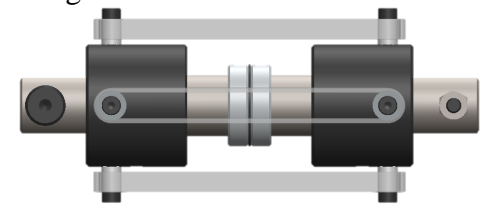

Controlled heating the LMPA part

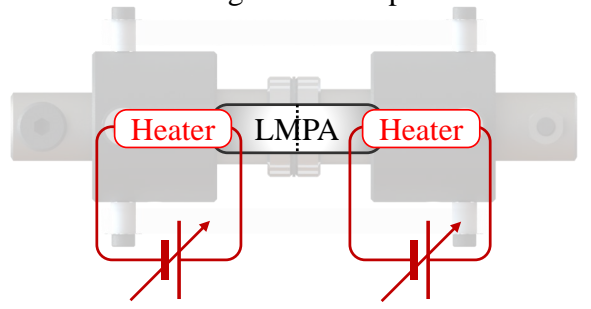

$\mathrm{C}$

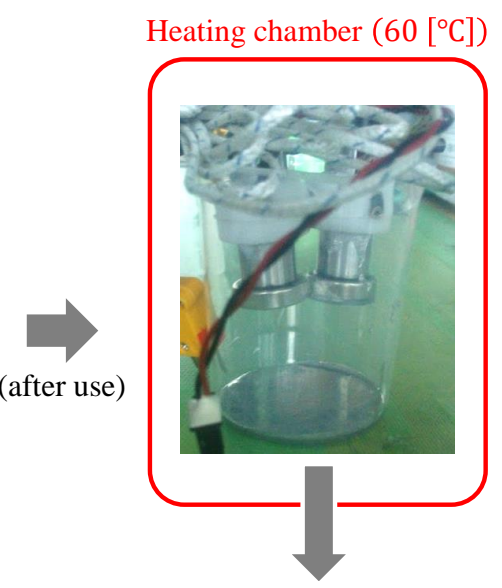

LMPA to stainless cup (recycled)

Figure 5: Manufacturing process and LMPA disposal process of the self-healing module. A) Liquid metal pouring. We poured the liquid metal into half of a self-healing module. The liquid metal had been melted in the stainless cup with a soldering iron. The photos show the half module with an empty state and the one filled with LMPA. B) Module assembly. First, we combined the half modules filled with LMPA using the magnetic force. Second, we attached the rubber bands to the module assembly. Third, we heated the inner LMPA component using the heaters. Actually, The third process is identical to the temperature control of the self-healing sequence. C) After being engaged in a robot, the module's LMPA was recycled. We melt the LMPA component in the module using the heating chamber whose temperature reference was $60\left[{ }^{*} \mathrm{C}\right]$. After the melting, the LMPA remnant was moved into the stainless cup again. 

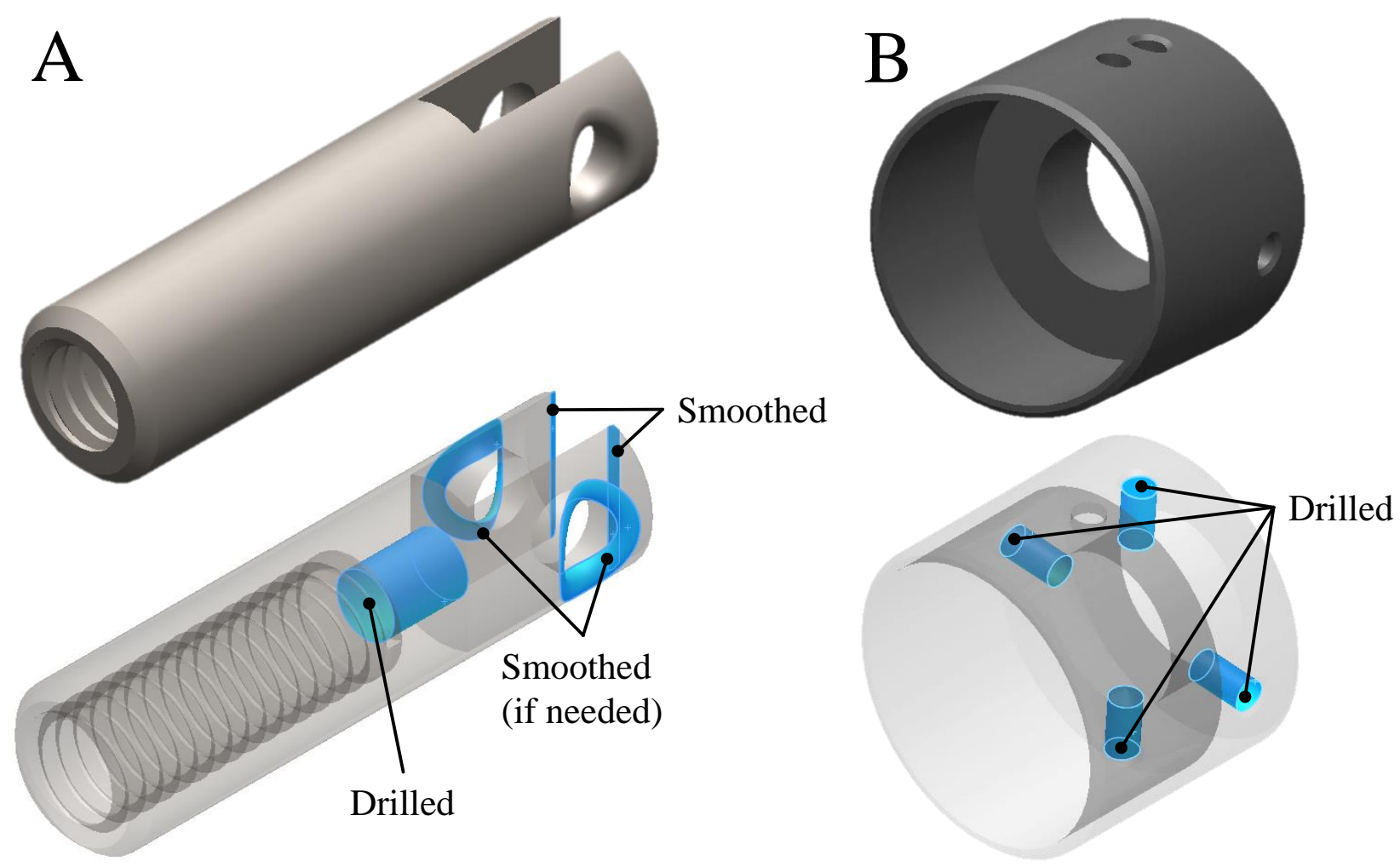

Figure 6: Manual processing of the self-healing module's off-the-shelf mechanical components. A) Link bar. We drilled it for installing the heater. Additionally, we smoothed the bar with power tools. Smoothing is necessary if the tendon wire contacts the part. B) Counterbore washer. We drilled four holes to attach the rubber band to the part. 

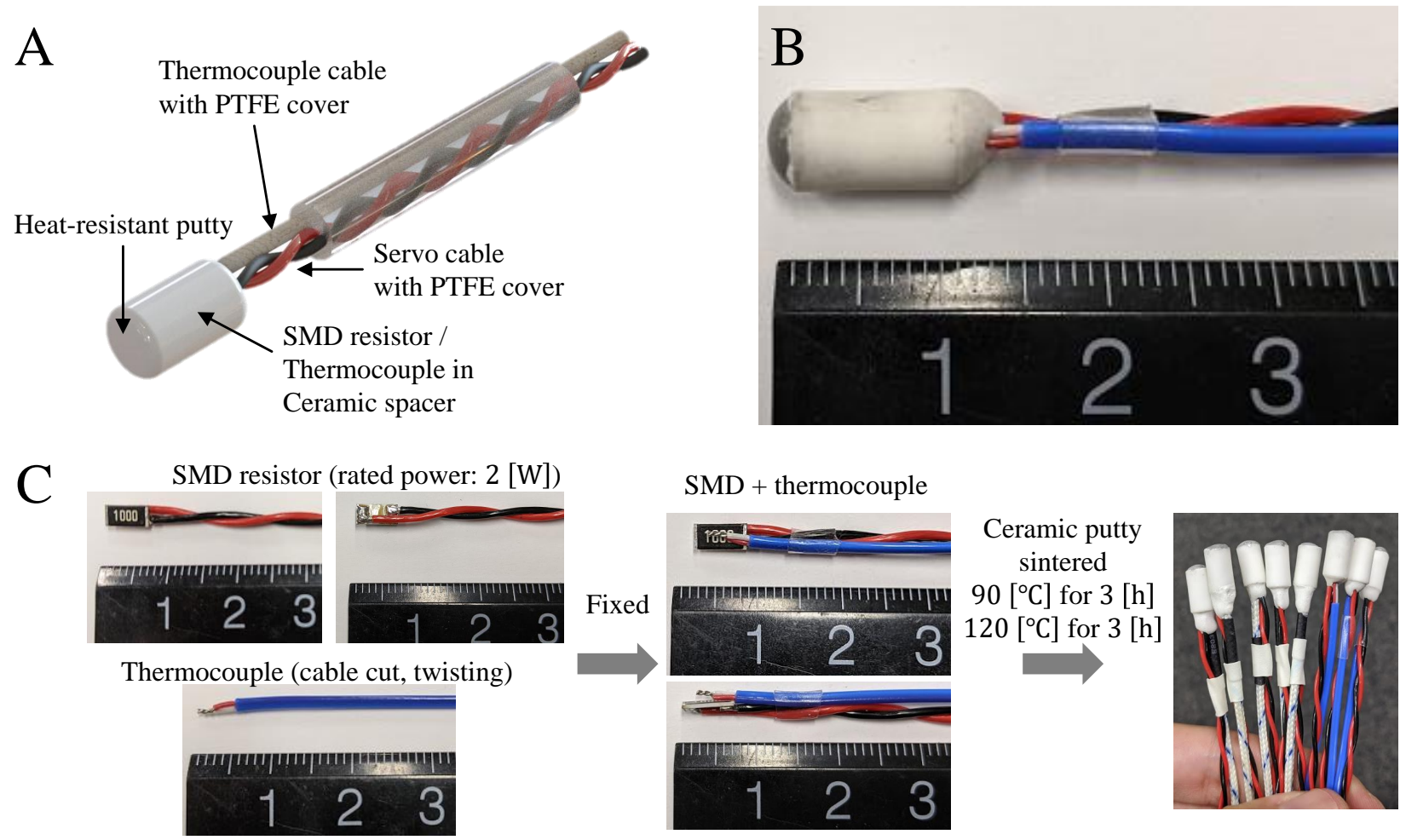

Figure 7: Heater unit for a self-healing module. A) components for the heater. The heater features the ease of customizing, temperature sensing, and mechanical strength which is indispensable for system integration. B) prototype model. The diameter is $6[\mathrm{~mm}]$, and the length is $10[\mathrm{~mm}]$. C) manufacturing process. The SMD resistor and the thermocouple were combined with the heat-shrinking tube. We used a heating chamber to sinter the ceramic putty which fills the resistor and the thermocouple. We build the heater in a scalable manner, which would be beneficial for prototyping and robot integration. 


\section{w/o vibration}

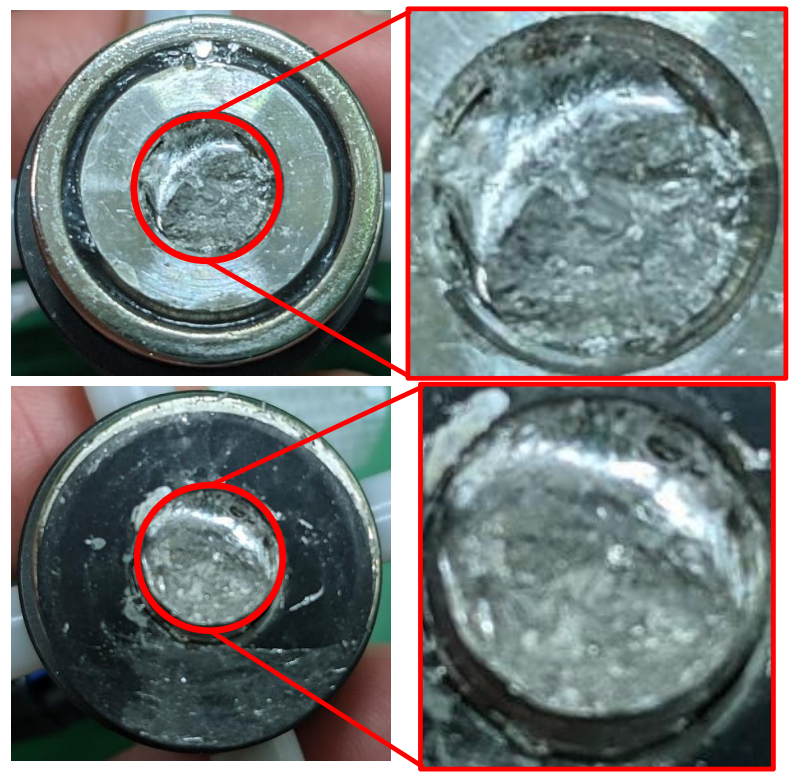

Amplitude: 10 [mm]

Frequency: $6[\mathrm{~Hz}]$

Cycles: 50
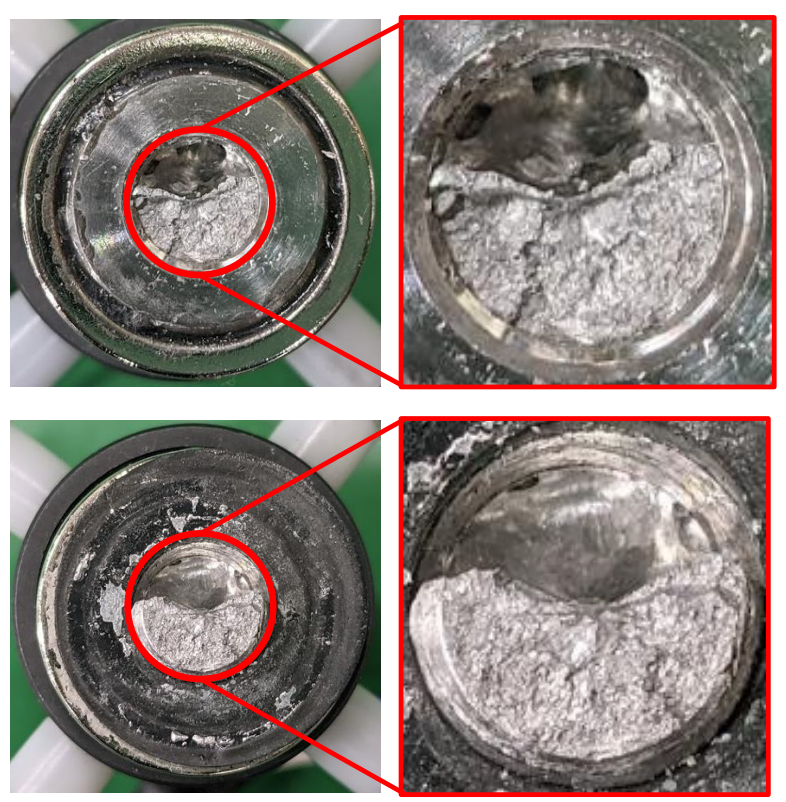

Figure 8: Fracture surface after the five cycles of tensile tests.

\section{w/o vibration}
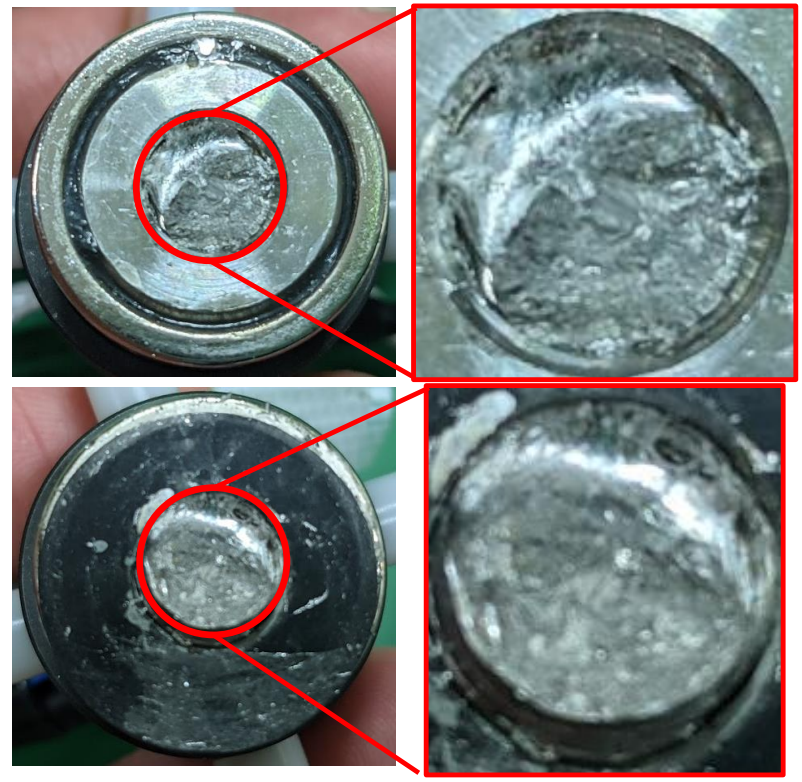

Amplitude: $10[\mathrm{~mm}]$

Frequency: $3[\mathrm{~Hz}]$

Cycles: 100

Figure 9: Fracture surface after the five cycles of tensile tests. 

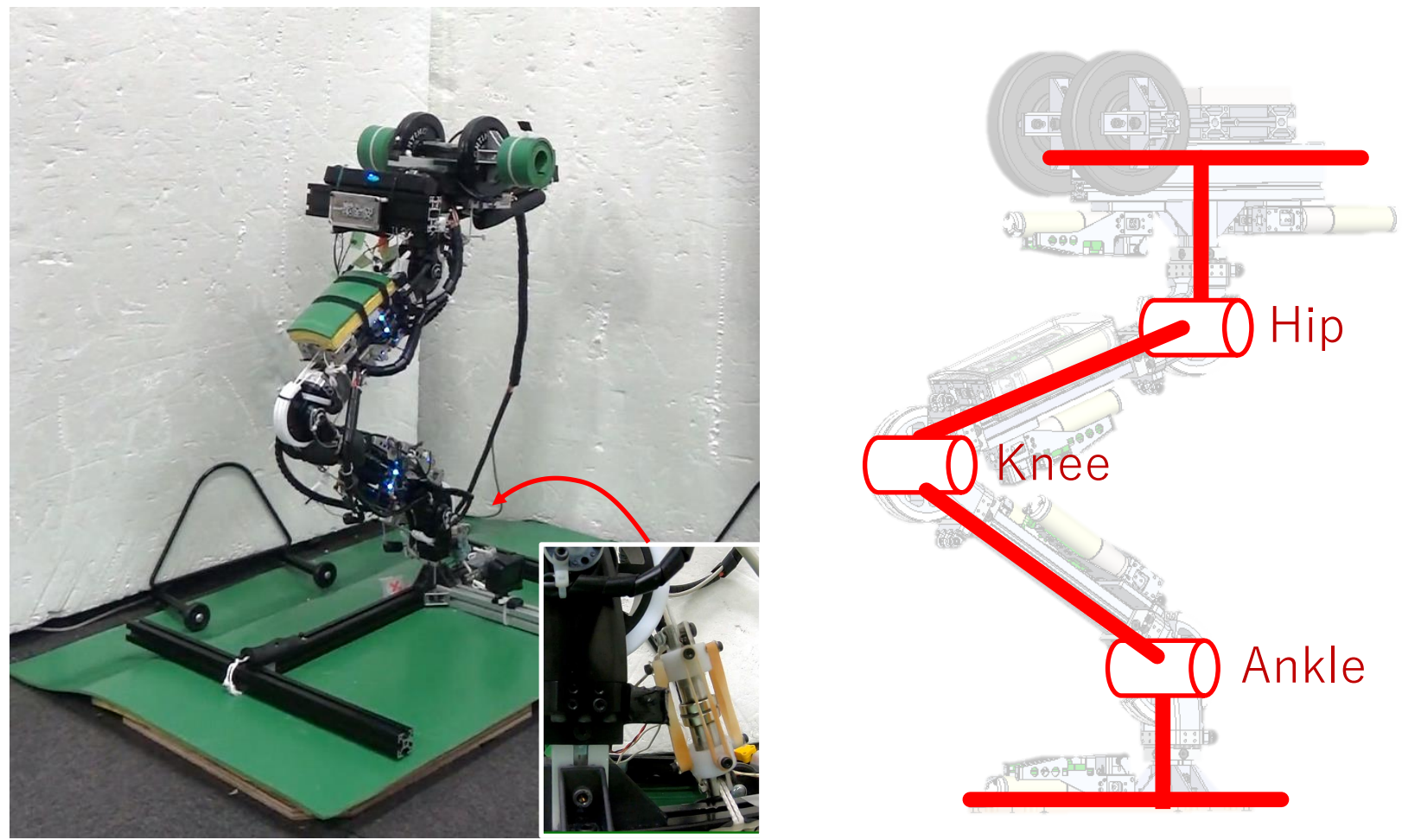

Figure 10: Tendon-driven legged robot prototype. The robot has three tendon-driven joints, and the ankle extensor wire is equipped with the proposed module.
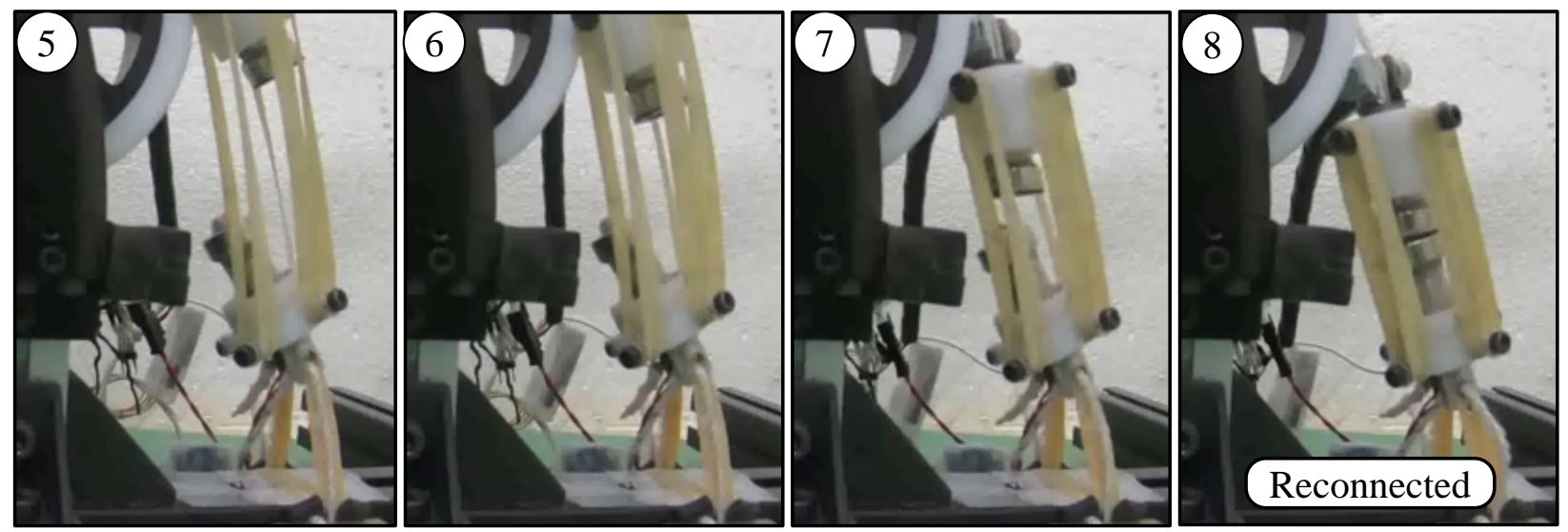

Figure 11: Snapshot of recovery motion. Interval time is 200 [ms]. Although the LMPA parts detached, the rubber bands generated restoring force to make them retouch again. 

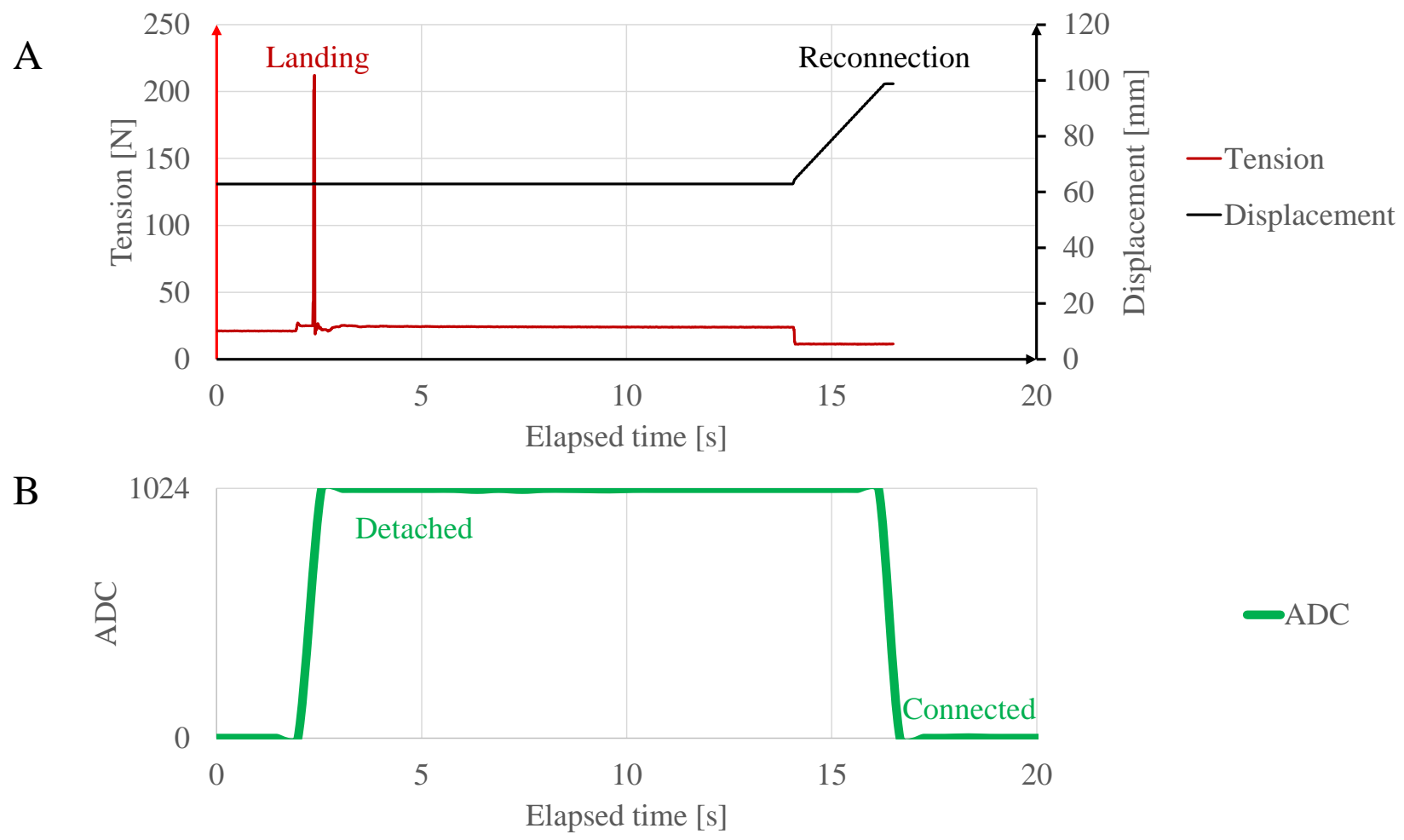

Figure 12: Result of the robot's landing motion. A) Tension and displacement plot of the ankle extensor during the landing experiment. The landing impact shows only 200 [N], which is due to the robot's relatively long sampling time of $8[\mathrm{~ms}]$. 

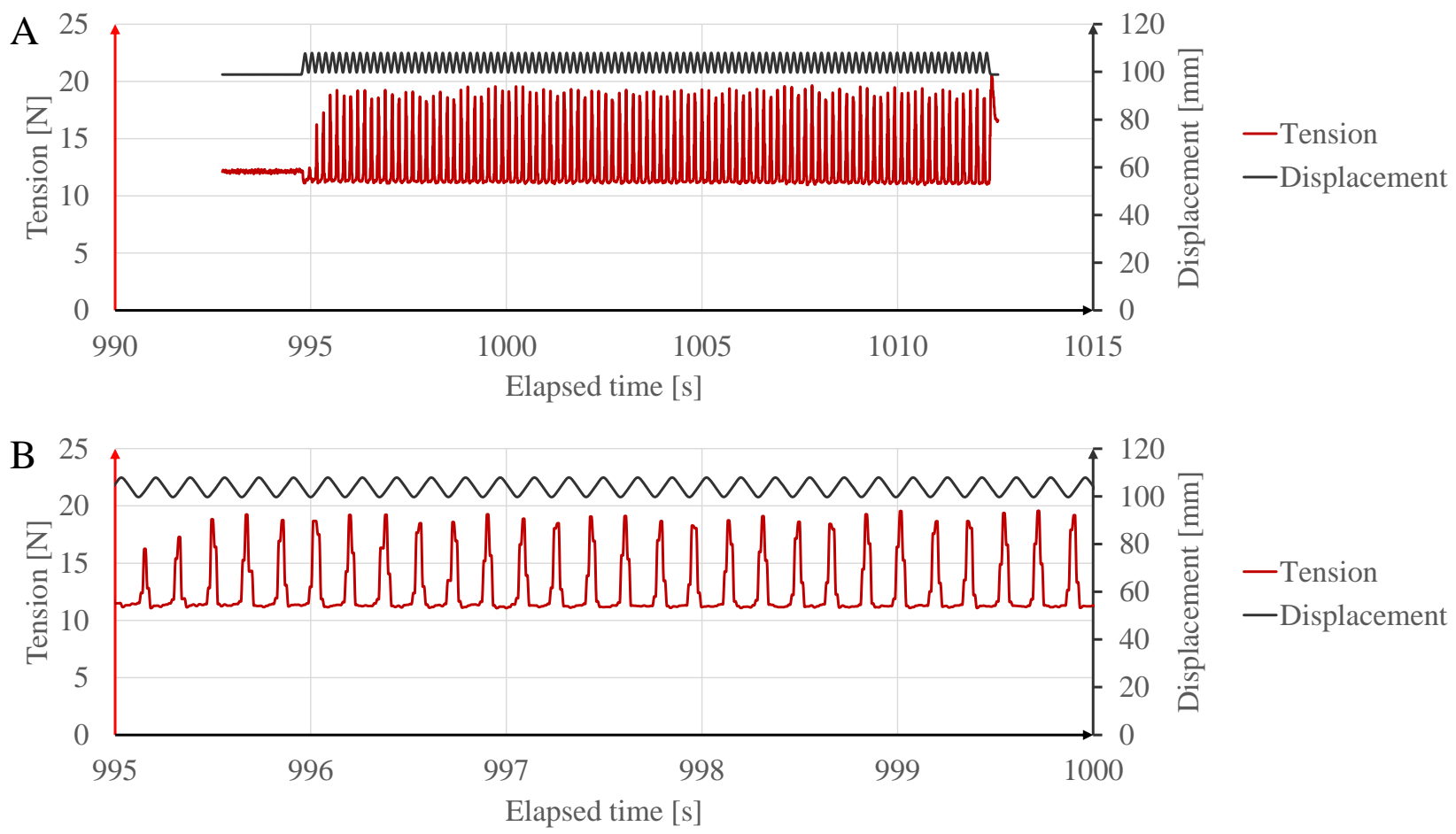

Figure 13: A) Tension plot during sloshing experiment. The vibration lasts for over 15 [s]. B) Enlarged view of $\mathrm{A}$ ). The tension is up to $20[\mathrm{~N}]$. The self-healing module's magnets and rubber bands kept the liquid metal from leak during the vibration. 

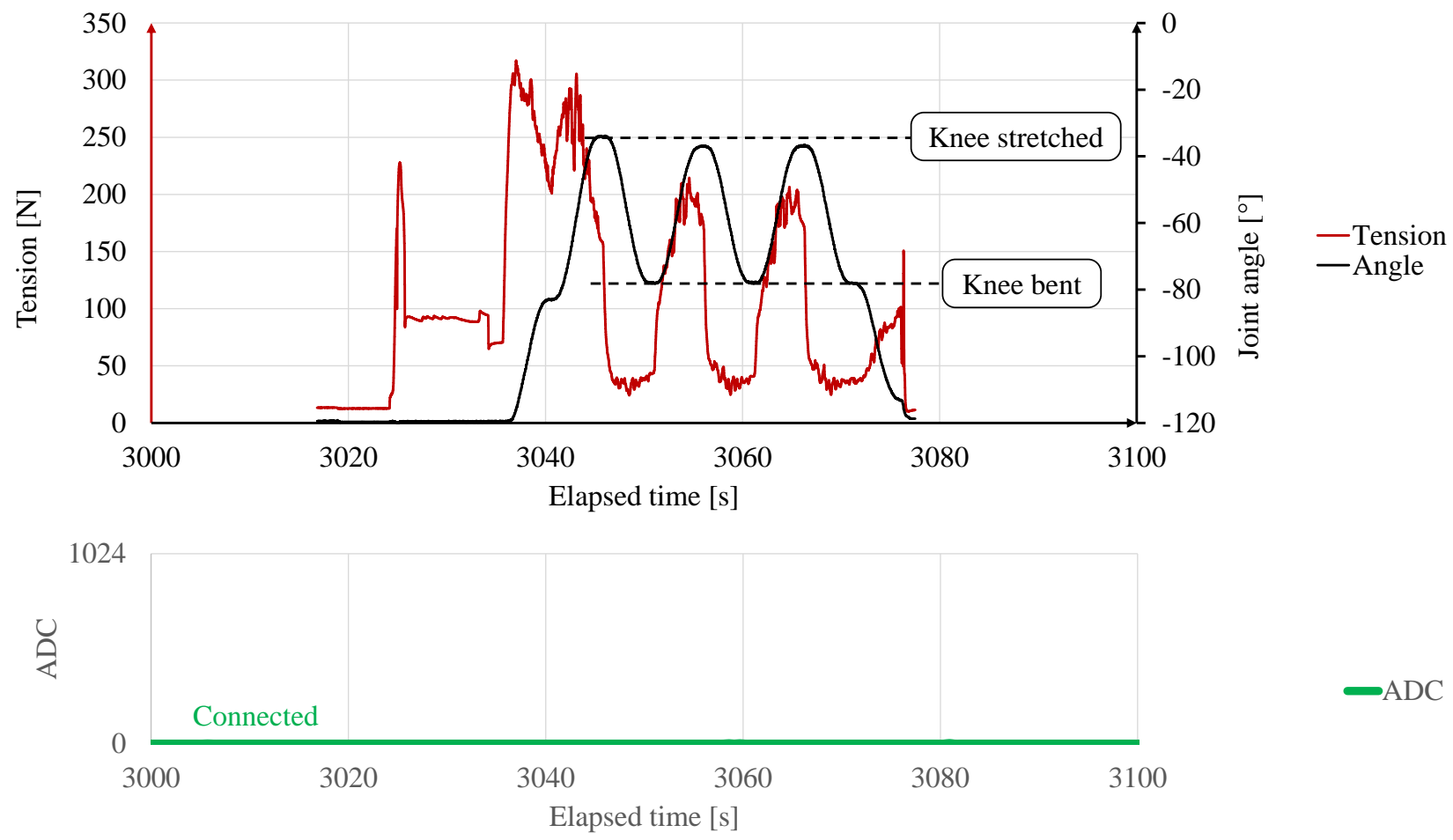

Figure 14: Result of the robot's squat behavior after the self-healing sequence. A) The ankle extensor's tension and the ankle joint's angle. The robot performed three times of squat motions after the self-healing sequence. The tension value was up to $400[\mathrm{~N}]$. B) The ADC value shows 0 due to the connection of the self-healing module. That is, the module never broke during the robot's squat motions. 


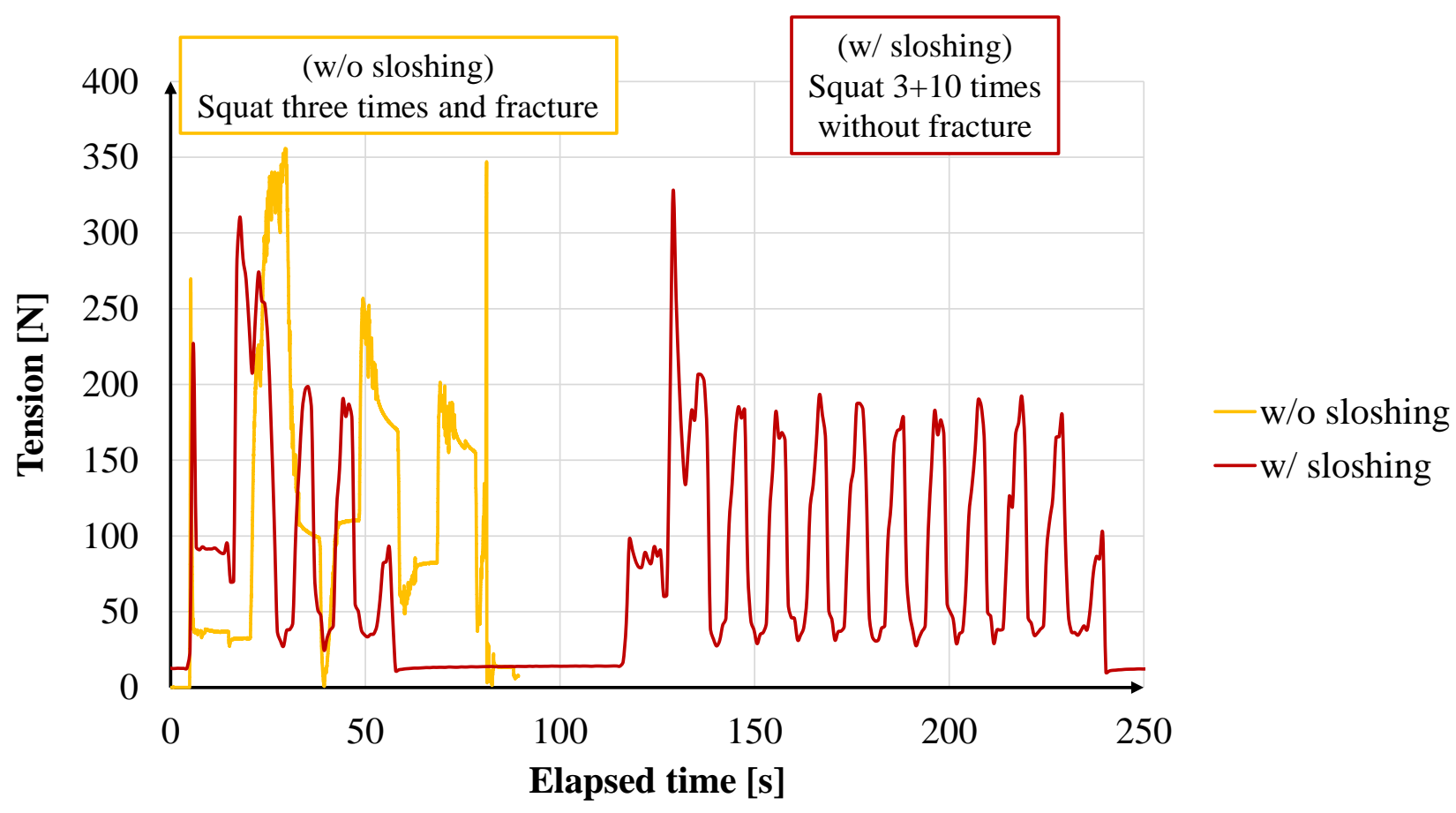

Figure 15: Tension comparison of the squat experiments. Without the sloshing, the robot was not able to finish the squat motion [1]. Without the sloshing, the robot was able to finish the squat motion successfully. 


\section{Supplementary Videos}

Rich media available at https://drive.google.com/file/d/1kT-B7NFQ6FlWHOp8sqimeDJ3hB5eJlsv/ view?usp=sharing

Rich media available at https://drive.google.com/file/d/1RJ9XIWvF1urlGaXytxuGsEIM-9E_2iB8/ view?usp=sharing

Rich media available at https://drive.google.com/file/d/10nm5L6rcrlnPS_IFj15GfPMXIX2Aw7in/ view?usp=sharing

Rich media available at https://drive.google.com/file/d/1De4N6zau16cjkF_lyubUZBfMM1-eqktS/ view?usp=sharing

Rich media available at https://drive.google.com/file/d/1zyA2kxh-nuG6sWCnCiDaRr5ILvSJje43/ view?usp=sharing

Rich media available at https://drive.google.com/file/d/1pCXopWxKMjdhvmVopqVVuYUuUG8wbEjQ/ view?usp=sharing

\section{Acknowledgements}

The authors acknowledge Dr. Takuma Shirai and Mr. Manabu Nishiura for their technical contributions.

\section{Conflict of interest}

The authors declare no conflict of interest.

\section{References}

Shinsuke Nakashima, Takuma Shirai, Yuki Asano, Yohei Kakiuchi, Kei Okada, and Masayuki Inaba. Resistance-based self-sensing system of active self-melting bolt towards autonomous healing structure. In 2018 IEEE International Conference on Soft Robotics (RoboSoft), pages 88-93, 2018. doi: 10.1109/ROBOSOFT.2018.8404902.

Shinsuke Nakashima, Takuma Shirai, Kento Kawaharazuka, Yuki Asano, Yohei Kakiuchi, Kei Okada, and Masayuki Inaba. An Approach of Facilitated Investigation of Active Self-healing Tension Transmission System Oriented for Legged Robots. In 2019 IEEE/RSJ International Conference on Intelligent Robots and Systems (IROS), pages 2567-2572, 2019. doi: 10.1109/IROS40897.2019.8967949.

Shinsuke Nakashima, Kento Kawaharazuka, Manabu Nishiura, Yuki Asano, Yohei Kakiuchi, Kei Okada, Koji Kawasaki, and Masayuki Inaba. Restoring Force Design of Active Self-healing Tension Transmission System and Application to Tendon-driven Legged Robot. In 2021 IEEE International Conference on Robotics and Automation (ICRA), pages 7033-7038, 2021. doi: 10.1109/ICRA48506.2021.9561531. 